\title{
Executive Dysfunction and Depression in Pediatric Temporal Lobe Epilepsy: The Contribution of Hippocampal Sclerosis and Psychosocial Factors
}

\author{
William A. Schraegle, ${ }^{1,2}$ Nancy L. Nussbaum, ${ }^{1,2}$ AND Jeffrey B. Titus ${ }^{1,3,4}$ \\ ${ }^{1}$ Comprehensive Epilepsy Program, Dell Children's Medical Center of Central Texas, Austin, Texas \\ ${ }^{2}$ Department of Educational Psychology, The University of Texas at Austin, Austin, Texas \\ ${ }^{3}$ Department of Neurology, Dell Medical School, The University of Texas at Austin, Austin, Texas \\ ${ }^{4}$ Department of Psychology, The University of Texas at Austin, Austin, Texas
}

(Received September 12, 2017; Final Revision January 29, 2018; Accepted January 31, 2018; First Published Online March 25, 2018)

\begin{abstract}
Objectives: Temporal lobe epilepsy (TLE) has been identified as a risk factor for increased depression features in children and adolescents; however, less is known regarding specific neurocognitive predictors of depression in this population above and beyond seizure-specific and sociodemographic factors. Methods: The study included 62 patients with TLE (64\% male) aged 8 to 16 years $(M=12.62 ; S D=2.26)$ who underwent comprehensive neuropsychological evaluation. Results: Correlation analyses revealed significant association between patient depression and WCST Total Perseverations, BRIEF Behavioral Regulation Index (BRI) and family stress. Perseverative errors on the WCST and the BRI were found to significantly predict depression features in youth with TLE. Patient performance on WCST was also found to fully mediate the significant relationship between hippocampal sclerosis (HS) and depression in pediatric TLE. Finally, logistic regression indicated HS in the presence of TLE was associated with a four-fold risk of clinically significant depression ratings. Conclusions: The current findings offer strong support for the relationship between executive function (EF) and depression in pediatric TLE. Also, as HS is not modifiable, these findings suggest EF intervention may be a potential modality for improving health-related quality of life (HRQOL) in youth with TLE. (JINS, 2018, 24, 606-616)
\end{abstract}

Keywords: Epilepsy, Temporal lobe, Executive function, Depression, Hippocampal sclerosis, Pediatric

\section{INTRODUCTION}

Children with epilepsy are at greater risk for psychopathology when compared with children in the general population (Davies, Heyman, \& Goodman, 2003; Rutter, Graham, \& Yule, 1970) and children with other neurological and chronic medical conditions (Rodenburg, Stams, Meijer, Aldenkamp, \& Deković, 2005). Depression is a common clinical feature observed in pediatric epilepsy, with prevalence estimates ranging from 20 to $40 \%$ (Ekinci, Titus, Rodopman, Berkem, \& Trevathan, 2009). Among children and adolescents with epilepsy, patients with temporal lobe epilepsy (TLE), the most common form of focal epilepsy, have higher rates of depression features (Salpekar et al., 2013; Schraegle \& Titus, 2017a; Titus, Kanive, Sanders, \& Blackburn, 2008), and recent

Correspondence and reprint requests to: Jeffrey B. Titus, Pediatric Neuropsychology, Dell Children's Medical Group, 1600 West 38th Street, Suite 320, Austin, TX 78731. E-mail: jbtitus@ascension.org research by Schraegle and Titus (2017a) demonstrated that children with TLE incurred over a two-fold risk for depression features. Because it is often under-recognized and undertreated in pediatric TLE (Kavanaugh, Scarborough, \& Salorio, 2015), depression in this population can contribute to psychosocial dysfunction and poorer health-related quality of life (HRQOL) (Plioplys, 2003; Schraegle \& Titus, 2017a).

The underlying mechanisms that contribute to depression in pediatric TLE are not well understood, but they are thought to involve multiple factors, spanning neurocognitive, sociodemographic, and seizure-specific influences. It has been posited that TLE and depression in adults share a similar neuropathogenic process (Gilliam et al., 2007; Richardson et al., 2007) with imaging investigations describing similar disruptions to the hippocampus, amygdala, and long-range frontal lobe projections (Keller, Baker, Downess, \& Roberts, 2009; Sheline, 2003). Not surprisingly, hippocampal abnormalities, such as hippocampal sclerosis (HS) (i.e., severe gliosis and cell loss in the hippocampus), in both adult TLE 
and depression are key contributors to frontotemporal network dysfunction (Focke et al., 2008; Kaiser, Andrews-Hanna, Wager, \& Pizzagalli, 2015). However, despite these overlapping neural substrates, the presence of cognitive risk factors for depression in pediatric epilepsy has received only limited attention to date.

Within the broader population of pediatric depression, deficits in executive function $(\mathrm{EF})$ are frequently reported. For example, youth with major depressive disorder (MDD) demonstrate deficits in phonemic fluency (Cataldo, Nobile, Lorusso, Battaglia, \& Molteni, 2005; Klimkeit, Tonge, Bradshaw, Melvin, \& Gould, 2011), set-shifting/cognitive flexibility (Holler, Kavanaugh, \& Cook, 2014), and auditory attention and working memory (Klimkeit et al., 2011). A recent meta-analysis by Wagner, Müller, Helmreich, Huss, and Tadić (2015) highlighted deficits associated with pediatric MDD in immediate and delayed verbal recall, sustained attention, inhibitory control, semantic fluency, and planning ability. Importantly, impaired behavioral regulation has been identified as a central feature of both adult MDD (Gyurak, Goodkind, Kramer, Miller, \& Levenson, 2012; Ladouceur et al., 2005) and TLE (Espinosa et al., 2010).

Adults with TLE have been found to have EF deficits in cognitive flexibility, abstraction, set-shifting, and problem solving (Cocoran \& Upton, 1993; Hermann, Seidenberg, \& Bell, 2002; Igarashi et al., 2002), and emerging research in pediatric TLE has demonstrated EF deficits in working memory (Gottlieb, Zelko, Kim, \& Nordli, 2012), sustained attention (Lopes, Simoes, Robalo, Fineza, \& Gonçalves, 2010; Rzezak et al., 2007), and cognitive flexibility (Igarashi et al., 2002; Rzezak et al., 2009). Rzezak et al. (2009) reported that $77 \%$ of youth with TLE had EF deficits on the Wisconsin Card Sorting Test (WCST). With exception to youth with frontal lobe epilepsy (FLE), youth with TLE exhibit a higher rate of EF impairment than youth with generalized epilepsy or focal epilepsy from other regions of the brain (Rzezak, Valente, \& Duchowny, 2014).

Corcoran and Upton (1993) postulated that HS in adult TLE is likely a key contributor to frontotemporal network dysfunction which is thought to underlie executive deficits. More recent neuroimaging studies in adult TLE have correlated executive performance to diminished left dorsal prefrontal cortex and left hippocampal volumes (Keller et al., 2009) and shown diminished left frontal activation on a verbal working memory task in patients with left HS (Campo et al., 2009). While the relationship between executive dysfunction and HS has been identified in adults, this relationship has yet to be fully examined in the pediatric TLE literature.

Additional seizure-specific variables are also believed to contribute to the risk for depression in youth with epilepsy, but previous literature has been equivocal about the role of many of the most commonly reported clinical features. For example, the evidence linking seizure frequency, age at seizure onset, and number of anti-epileptic medications with depression is mixed (Austin et al., 2001; Thome-Souza et al., 2004), although longer duration of epilepsy may have a somewhat stronger association (Austin et al., 2001;
Oguz, Kurul, Dirik, \& Eylül, 2002). The nature of these relationships has not been well examined in the pediatric literature, and there is no information about whether risk factors remain consistent throughout development. Additionally, despite evidence in the adult literature of a relationship between epilepsy-related brain pathology and depression, the pediatric literature has not examined such associations. For example, Gilliam et al. (2007) identified HS as a significant risk factor for depression in adults with TLE; however, no studies to date consider whether HS increases the risk for psychopathology in youth with TLE.

Sociodemographic risk factors have long been shown to be a risk-factor for depression in the general population, and the same is true in the context of epilepsy. For instance, previous research has shown that up to $50 \%$ of patients with epilepsy and depression have a family history of depression (Plioplys, 2003). Thome-Souza et al. (2004) reported that a family history of psychopathology increases the risk of depression in younger children. Similarly, higher family stress has been found to correlate with increased behavior problems in youth, even when controlling for seizure variables (Ekinci et al., 2009).

\section{Study Aims}

Despite the known clinical relationship between pediatric TLE and depression, no studies to date have explored neurocognitive, sociodemographic and seizure-specific vulnerabilities in this population. Therefore, the current study examined the following research questions: (1) Are there cognitive risk factors for depression in pediatric epilepsy? (2) Do cognitive risk factors contribute uniquely to depression in pediatric epilepsy, or is it better explained by sociodemographic or seizure-specific factors? (3) Is HS associated with EF deficits in youth with TLE? (4) Does the presence of HS incur greater risk for depression in children and adolescents with TLE?

\section{METHODS}

\section{Participants}

Youth between the ages of 8 and 16 years with a prior diagnosis of TLE who were referred for epilepsy treatment at a tertiary care health system were eligible for inclusion. Children were referred for neuropsychological services by their treating neurologist or epileptologists based upon clinical need. Specifically, approximately $42 \%$ of the sample was referred as candidates for surgery to treat intractable seizures. The remainder of the sample was composed of children with TLE who were experiencing cognitive difficulties that would warrant a neuropsychological evaluation. TLE was confirmed by board-certified epileptologists via clinical evaluation, ictal electroencephalograph (EEG) monitoring, and concordant interictal EEG findings, consistent with International League Against Epilepsy criteria (Engel, 2001). Seizure laterality was also investigated; however, those children with bilateral epileptiform activity $(n=2)$ were excluded due to incomplete 
data. HS was identified by magnetic resonance imaging (MRI), namely increased signal intensity on T2-weighted and FLAIR images, and verified by a pediatric neuroradiologist.

Approval by the Seton Institutional Review Board and written consent by parents were obtained to access archival data. Exclusion criteria included the presence of extratemporal epileptiform activity on EEG, previous neurosurgery, diagnosis of hearing or visual impairment, or a positive history of psychosis. Children were also excluded if caregivers could not speak or read English.

\section{Sociodemographic and Epilepsy-Specific Variables}

Demographic and seizure-related variables were taken directly from a combination of prior medical records, parent interview, and a standardized family information questionnaire completed by a parent the day of evaluation. The following demographic data were collected: participant age, gender, ethnicity, maternal education level, parental marital/relationship status, and family stress (i.e., "Please rate the overall degree of you family's stress in the past year"; coded: $1=$ very little stress, $2=$ lower than typical, 3 =typical stress, $4=$ higher than typical, and $5=$ very high stress).

Parental history of psychopathology was coded as 1 (positive identification) or 0 (no history identified). Parental psychiatric history included depressive disorders, anxiety disorders, and bipolar disorders. Seizure-related variables included the following: age at seizure onset, number of epilepsy-related medications (antiepileptic drugs [AEDs]), number of failed AEDs, seizure frequency (coded: $0=$ never,
$1=$ yearly, $\quad 2$ =quarterly, $3=$ monthly,$\quad 4=$ weekly, and $5=$ daily), duration of epilepsy (i.e., time since the first seizure), and positive identification of HS (coded: $0=$ negative and $1=$ positive identification).

\section{Neuropsychological and Behavioral Measures}

All children and adolescents were administered neuropsychological tests by individuals trained in standardized testing procedures. Neuropsychological variables used in the present study are outlined in Table 1. Additionally, caregiver ratings on the Behavior Assessment for Children-2 (BASC-2) are defined as follows: T-scores at or below 59 are considered in the "Normal" range; T-scores of 60-69 are considered "At-Risk" and indicate behaviors that may warrant clinical attention; and T-scores $\geq 70$ suggest "Clinically Significant" levels and represents a high level of maladjustment warranting clinical attention (Reynolds \& Kamphaus, 2004).

\section{Statistical Analysis}

To better characterize how sociodemographic sample features relate to depression, chi-square tests were conducted on demographic variable frequencies at three levels of depression severity (i.e., Normal, At-Risk, and Clinically Significant). Variables were dichotomized as follows: maternal education (less than high school $v s$. high school and beyond), parent relationship status (divorced/separated vs. married/ together), parental psychiatric history (positive $v s$. negative),

Table 1. Overview of neuropsychological and behavioral measures

\begin{tabular}{|c|c|c|c|}
\hline Domain & Ability & Measure/test & \\
\hline \multirow[t]{5}{*}{ Language } & Expressive Vocabulary & Confrontational Naming & $\mathrm{BNT}^{\mathrm{a}}$ \\
\hline & Lexical Knowledge & Vocabulary & WISC-IV ${ }^{b}$ \\
\hline & Abstract Verbal Reasoning & Similarities & WISC-IV \\
\hline & Verbal Fluency, Phonemic & Letter Fluency & DKEFS $^{c}$ \\
\hline & Verbal Fluency, Semantic & Category Fluency & DKEFS \\
\hline \multirow[t]{2}{*}{ Visuospatial } & Perceptual Organization & Block Design & WISC-IV \\
\hline & Abstract Spatial Reasoning & Matrix Reasoning & WISC-IV \\
\hline \multirow[t]{3}{*}{ Memory } & Verbal & List: $1-5$ Total, SDFR, LDFR & CVLT-C $^{\mathrm{d}}$ \\
\hline & & Narrative: MFS, MFSD & TOMAL- $2^{\mathrm{e}}$ \\
\hline & Nonverbal & Facial Memory & TOMAL-2 \\
\hline \multirow[t]{5}{*}{ Executive } & Flexibility/Problem Solving & Perseverative Errors & $\mathrm{WCST}^{\mathrm{f}}$ \\
\hline & Immediate Attention & Digits Forward & WISC-IV \\
\hline & Working Memory & Digits Backward & WISC-IV \\
\hline & Metacognition & Metacognitive Index & BRIEF-Parent Form ${ }^{\mathrm{g}}$ \\
\hline & Behavioral Regulation & Behavioral Regulation Index & BRIEF-Parent Form \\
\hline Behavior & Depressive Features & Depression subscale & BASC-2-Parent Form ${ }^{\text {h }}$ \\
\hline
\end{tabular}

${ }^{\mathrm{a} B}$ Boston Naming Test (Kaplan, Goodglass, Weintraub, 2001).

${ }^{\mathrm{b}}$ Wechsler Intelligence Scale for Children-Fourth Edition (Wechsler, 2003).

${ }^{\mathrm{c}}$ Delis-Kaplan Executive Function System (Delis, Kaplan, Kramer, 2001).

${ }^{\mathrm{d} C}$ California Verbal Learning Test-Children's Version (Delis, Kramer, Kaplan, Ober, 1994).

${ }^{\mathrm{e}}$ Test of Memory and Learning, $2^{\text {nd }}$ Edition (Reynolds \& Voress, 2007).

${ }^{f}$ WCST: Wisconsin Card Sorting Test (Heaton, Chelune, Talley, Kay, \& Curtiss, 1993).

${ }^{\mathrm{g} B e h a v i o r ~ R a t i n g ~ I n v e n t o r y ~ o f ~ E x e c u t i v e ~ F u n c t i o n: ~ P a r e n t ~ R a t i n g ~ S c a l e ~(G i o i a, ~ I s q u i t h, ~ G u y, ~ a n d ~ K e n w o r t h y, ~ 2000) . ~}$

${ }^{\text {h} B e h a v i o r ~ A s s e s s m e n t ~ S y s t e m ~ f o r ~ C h i l d r e n ~-~ 2: ~ P a r e n t ~ R a t i n g ~ S c a l e ~(R e y n o l d s ~ \& ~ K a m p h a u s, ~ 2004) . ~}$ 
and family stress ("higher than typical stress" and "very high stress" vs. $\leq$ "typical stress" ratings). Depression severity was then examined within the following variables: maternal education less than high school, parents divorced or separated, positive parental psychiatric history, and family stress $\geq$ "higher than typical."

All neuropsychological measures were converted to age-corrected standard scores using the best available norms. Correlations (i.e., Pearson, point biserial, as appropriate) were conducted to identify significant demographic and seizure-specific variables for statistical control as well as to characterize the associations between neuropsychological variables and the BASC-2 depression subscale. Next, a simultaneous linear regression was conducted to determine unique predictors of depression. Multicollinearity was tested and found not to be present as predictor inflation factor (variance inflation factor) and collinearity tolerance were within acceptable limits. Partial correlations were also examined to consider shared variance and the unique contribution of each variable to depression.

Three separate analyses of variance (ANOVAs) were conducted to test the main effect of HS. Mediation was assessed using both the traditional causal steps approach and non-parametric bootstrapping procedures (Preacher \& Hayes, 2004), and a binary logistic regression was conducted to determine whether HS served as a specific risk factor for depression.

Because of the exploratory nature of the study and the need to balance Type I and Type II error, the olevel for all significance tests was set at 0.05 a priori. However, only moderate-to-large correlations $(\mathrm{r} \geq|0.32| ; p \leq .01)$ were interpreted as evidence of significant associations between variables. All analyses were conducted using SPSS version 21.0 (SPSS Inc., Chicago IL).

\section{RESULTS}

\section{Sample Characteristics}

Table 2 lists sociodemographic and seizure-specific sample characteristics. The study included 62 children and adolescents with TLE (64\% male) aged 8 to 16 years $(M=12.62$; $S D=2.26$ ). Children and adolescents came from various ethnic backgrounds, with 45\% Caucasian, 26\% African American, 19\% Hispanic, and less than 10\% identifying as Asian or Polynesian. Around 58\% of the sample came from divorced or separated households, with the majority of mothers attending at least "some college." Positive or ongoing psychiatric history in parents was identified in 58\% and overall family stress averaged between "typical stress" to "higher than typical stress."

Most patients experienced seizure onset in childhood $(M=6.69 ; S D=4.02)$, with roughly $16 \%$ of the sample reporting seizure onset in the first year of life. Seizure frequency occurred yearly in $15 \%$, quarterly in approximately $10 \%$, monthly in approximately $40 \%$, weekly in approximately $19 \%$, and daily seizures in $16 \%$. Medication regimen for children and adolescents averaged one current AED $(M=1.45 ; S D=0.69)$ and around one failed AED trial $(M=1.45 ; S D=1.55)$. HS was identified in 17 of patients $(27 \%)$, and $53 \%$ of youth in the sample had seizures lateralized to the left temporal lobe.

As seen in Table 3, neuropsychological performance predominantly fell within the low average range across domains. Parent rated depression features on the BASC-2 fell in the At-risk range as a group $(M=60.55 ; S D=14.38)$, and 21 children $(33.9 \%)$ were rated as having Clinically Significant features of depression. These rates are consistent with previous research (Salpekar et al., 2013; Schraegle \& Titus, 2017a; Titus et al., 2008).

\section{Sociodemographic Features by Depression Severity}

The percentages of various sociodemographic sample features grouped by depression severity (i.e., Normal, At-Risk, and Clinically Significant) are displayed in Figure 1. Group differences on depression severity were not identified for maternal education, parent relationship status, or parental psychiatric history $(p>.05)$. However, elevated levels of family stress were more prevalent in youth with clinically elevated levels of depression compared to those with at-risk ratings $\left(\chi^{2}=5.22 ; p=.022\right)$ and ratings falling in the normal range $\left(\chi^{2}=7.88 ; p=.005\right)$ (see Table 4$)$.

Table 2. Descriptive values for sociodemographic and epilepsy variables

\begin{tabular}{lc}
\hline \hline Characteristic & Mean $(S D)$ or $N(\%)$ \\
\hline$N$, (males and females) & $62(40$ and 22$)$ \\
Age (years) $(S D)$ & $12.62(2.26)$ \\
Racial background $N(\%$ minority) & $33(53.2)$ \\
Maternal education $N(\%)$ & - \\
$\quad$ <High school & $9(14.5)$ \\
High school & $16(25.8)$ \\
Some college & $23(37.1)$ \\
College & $11(17.7)$ \\
Graduate degree & $3(4.8)$ \\
Parents separated/divorced $N(\%$ yes $)$ & $36(58.1)$ \\
Parental psychiatric history $N(\%$ yes $)$ & $36(58.1)$ \\
Family stress $N(\%)$ & - \\
$\quad$ Very little & $1(1.6)$ \\
Lower than typical & $8(12.9)$ \\
Typical stress & $27(43.5)$ \\
Higher than typical & $18(29)$ \\
Very high stress & $8(12.9)$ \\
Age of seizure onset & $6.69(4.02)$ \\
Epilepsy duration (years) & $5.99(4.61)$ \\
Seizure frequency & $3.10(1.30)$ \\
No. of failed AEDs & $1.45(1.55)$ \\
No. of current AEDs & $1.45(0.69)$ \\
Surgical candidate/Phase $1 N(\%)$ & $26(41.9)$ \\
Hippocampal sclerosis $N(\%$ yes $)$ & $17(27.4)$ \\
Seizure laterality $N(\%$ left $)$ & $33(53.3)$ \\
\hline \hline &
\end{tabular}


Table 3. Neuropsychological profile of the current sample

\begin{tabular}{lc}
\hline \hline Domain & Mean $(S D)$ \\
\hline Language & \\
BNT Total Score & $72.88(29.58)$ \\
WISC-IV Vocabulary & $7.44(3.93)$ \\
WISC-IV Similarities & $7.69(3.06)$ \\
DKEFS Letter Fluency & $7.26(4.15)$ \\
DKEFS Category Fluency & $7.90(3.75)$ \\
Visuospatial reasoning & \\
WISC-IV Block Design & $7.46(3.54)$ \\
WISC-IV Matrix Reasoning & $8.09(3.35)$ \\
Verbal memory & \\
CVLT-C Total & $87.37(16.86)$ \\
CVLT-C Short-Delay Free Recall & $80.03(21.02)$ \\
CVLT-C Long-Delay Free Recall & $85.02(21.69)$ \\
TOMAL Memory for Stories & $8.40(3.20)$ \\
TOMAL Memory for Stories-Delayed & $7.71(3.31)$ \\
Nonverbal memory & \\
TOMAL Facial Memory & $7.44(3.11)$ \\
Attention and executive function & \\
WCST Perseverative Errors & $96.63(16.88)$ \\
Digits Forward & $6.66(3.08)$ \\
Digits Backward & $7.40(3.07)$ \\
BRIEF Behavioral Regulation Index & $61.94(13.15)$ \\
BRIEF Metacognitive Index* & $65.35(10.15)$ \\
Behavioral function & \\
BASC-2 Depression Scale ${ }^{*}$ & $60.55(14.38)$ \\
\hline \hline
\end{tabular}

Note. Standard score, mean $=100, S D=15$; T-score, mean $=50, S D=10$; scaled score, mean $=10, S D=3$. BRIEF and BASC ratings use T-scores $(*)$, and higher scores indicate greater levels of impairment.

BASC-2 = Behavior Assessment System for Children - 2: Parent Rating Scale; BNT $=$ Boston Naming Test; BRIEF $=$ Behavior Rating Inventory of Executive Function: Parent Rating Scale; CVLT-C =California Verbal Learning Test-Children's Version; DKEFS $=$ Delis-Kaplan Executive Function System; TOMAL = Test of Memory and Learning, $2^{\text {nd }}$ Edition; WCST $=$ Wisconsin Card Sorting Test; WISC-IV $=$ Wechsler Intelligence Scale for Children-Fourth Edition.

\section{Correlations Between Neuropsychological Variables and Depression Ratings}

Pearson's correlations between neuropsychological measures and parent BASC-2 depression ratings are presented in Table 5.
The results indicated significant associations between depression ratings and perseverative errors on the WCST $(r=-0.426$; $p<.001)$ and the BRIEF BRI $(\mathrm{r}=0.498 ; p \leq .0001)$. Moderate associations were indicated between depression ratings and CVLT-C Total Score $(\mathrm{r}=-0.258 ; \quad p=.043)$, CVLT-C Short-Delay Free Recall $(\mathrm{r}=-0.276 ; p=.030)$, WISC-IV Digits Forward $(\mathrm{r}=-0.270 ; p=.034)$, and metacognition on the BRIEF (MI; $\mathrm{r}=0.281 ; p=.027$ ), but these associations did not survive correction for multiple comparisons.

When examining sociodemographic and seizure-specific variables, the sole significant correlation was a positive association between family stress and depression ratings $(\mathrm{r}=0.377 ; p=.003)$. There was a trend for HS to be related to depression ratings $(\mathrm{r}=0.316 ; p=.012)$, but maternal education $(\mathrm{r}=-0.163)$, parental psychiatric history $(r=0.227)$, age of seizure onset $(r=-0.07)$, epilepsy duration $(r=0.091)$, seizure frequency $(r=-0.047)$, number of failed AEDs $(r=-0.071)$, and number of current AEDs $(r=0.124)$ were unrelated to depression ratings $(p>.05)$.

\section{Defining Specific Predictors of Depression in Pediatric TLE}

To determine whether EF variables contributed any unique variance to the prediction of youth depression ratings above and beyond family stress, a linear regression was conducted (Table 6). In an effort to maintain adequate power for the present analysis, predictors were chosen based on moderate-to-large associations with depression ratings $(p \leq .01)$. Partial and semipartial correlations were examined to explore the amount of variance uniquely accounted for by each predictor, thus providing an index of relative importance.

Using the BRIEF BRI variable, WCST Perseverative Score, and family stress variables to predict depression, the regression model was significant $\left(R^{2}=.326\right.$; adj $R^{2}=0.291 ; \mathrm{F}_{3,61}=9.35$; $p<.0001)$. When common variance among the three predictors was considered, the BRI (partial $\mathrm{r}=0.282 ; p=.029$ ) and WCST Perseverative Score (partial $\mathrm{r}=-0.263 ; p=.042$ ) both emerged as unique predictors of parent rated depression

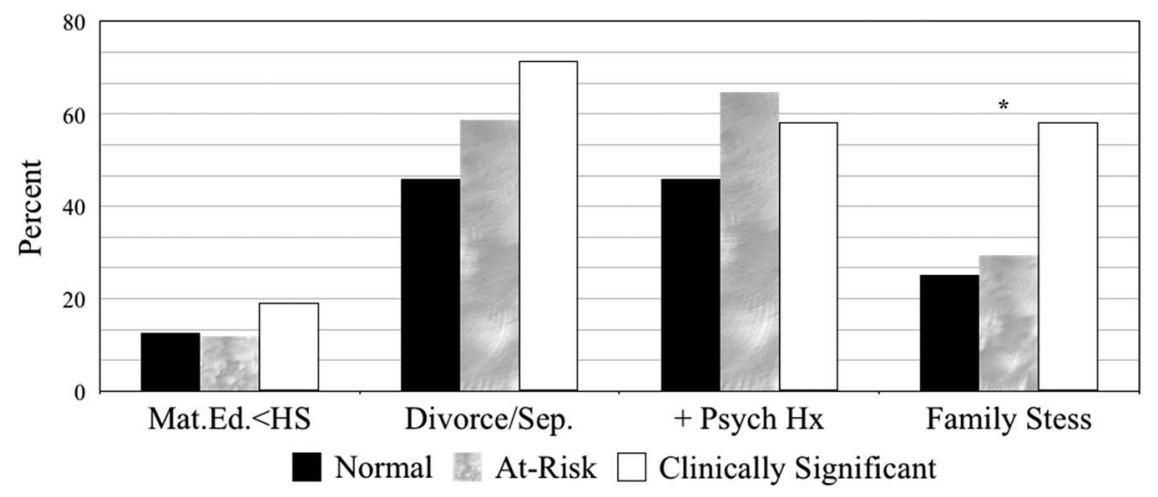

Fig. 1. Sociodemographic features by depression severity.

Note. Normal, T-scores $\leq 59$; at-risk, T-scores between 60-69; and clinically significant and T-scores $\geq 70$. Mat.Ed. $<$ HS $=$ Maternal education less than high school; Divorce/Sep.=Parents separated or divorced; + Psych $\mathrm{Hx}=$ Positive parental psychiatric history 
Table 4. Counts and percentages of sociodemographic features by depression severity

\begin{tabular}{|c|c|c|c|}
\hline \multirow[b]{2}{*}{ Sociodemographic variables } & \multicolumn{3}{|c|}{ Depression severity } \\
\hline & Normal $(N=24)$ & At-risk $(N=17)$ & Clinically elevated $(N=21)$ \\
\hline Maternal education $<$ high school & $3(12.5 \%)$ & $2(11.8 \%)$ & $4(19.0 \%)$ \\
\hline Parents separated or divorced & $11(45.8 \%)$ & $10(58.8 \%)$ & $15(71.4 \%)$ \\
\hline Family psychiatric history & $11(45.8 \%)$ & $11(64.7 \%)$ & $14(66.7 \%)$ \\
\hline Elevated family stress ${ }^{*}$ & $6(25.0 \%)$ & $5(29.4 \%)$ & $14(58.3 \%)$ \\
\hline
\end{tabular}

*Significant difference between clinically elevated and normal; $p<0.01$. Significant difference between clinically elevated and at-risk; $p<0.05$.

\section{Comparing the Impact of Hippocampal Sclerosis on Depression and Executive Funtion}

Because EF variables were found to significantly predict parent depression ratings, differences between youth with $(n=17)$ and without HS $(n=45)$ were examined on depression ratings, WCST scores, and the BRIEF BRI. Despite uneven group sample sizes, Levene's Test revealed the homogeneity of

Table 5. Bivariate correlations between neuropsychological variables and BASC-2 depression ratings

\begin{tabular}{lcc}
\hline \hline & $\begin{array}{c}\text { Correlation } \\
\text { coefficient }\end{array}$ & $p$-Value \\
Domain & & \\
\hline Language & -0.052 & .689 \\
BNT total raw score & -0.162 & .243 \\
Vocabulary & -0.091 & .511 \\
Similarities & -0.246 & .054 \\
DKEFS Letter Fluency & -0.278 & $.029^{*}$ \\
DKEFS Category Fluency & & \\
Visuospatial reasoning & -0.078 & .575 \\
Block Design & 0.045 & .748 \\
Matrix Reasoning & & \\
Verbal memory & -0.258 & $.043^{*}$ \\
CVLT-C Total & -0.276 & $.030^{*}$ \\
CVLT-C Short-Delay Free & -0.133 & .303 \\
CVLT-C Long-Delay Free & -0.132 & .307 \\
TOMAL Memory for Stories & -0.241 & .059 \\
TOMAL Memory for Stories- & & \\
Delayed & & \\
Nonverbal memory & -0.131 & .308 \\
TOMAL Facial Memory & & \\
Attention and executive & & $.027^{*}$ \\
functioning & -0.447 & $.001^{* * *}$ \\
WCST Perseverative Errors & -0.270 & $.034^{*}$ \\
Digits Forward & 0.498 \\
Digits Backward & & .329 \\
BRIEF Behavioral Regulation & & \\
Index & & \\
BRIEF Metacognitive Index & & \\
\hline \hline
\end{tabular}

BRIEF $=$ Behavior Rating Inventory of Executive Function: Parent Rating Scale; BNT $=$ Boston Naming Test CVLT-C $=$ California Verbal Learning Test-Children's Version; DKEFS $=$ Delis-Kaplan Executive Function System; TOMAL $=$ Test of Memory and Learning, $2^{\text {nd }}$ Edition; WCST $=$ Wisconsin Card Sorting Test.

* $p<.05$.

${ }^{* * *} p<.01$.

${ }^{* * *} p<.001$. variance assumption was met $(p>.10)$. For youth with a positive identification of HS, depression ratings were in the At-risk range as a group $(M=69.94 ; S D=14.79)$. WCST performance was in the low average range $(M=89.82$; $S D=17.62)$, and parent ratings of behavioral regulation on the BRIEF were in the normal range $(M=59.88 ; S D=12.87)$.

Those youth without HS had depression ratings in the normal range for their age $(M=58.20 ; S D=13.71)$, WCST Perseverative Errors Score in the average range $(M=99.20 ; S D=16.04)$, and behavioral regulation ratings at the upper limit of the normal range $(62.71 ; S D=13.31)$. Because no group differences were identified for seizurespecific variables, sociodemographic characteristics, or intellectual level $(p>.05)$, covariates were not used. ANOVA identified significant group differences for depression ratings $\left(F_{1,61}=6.63 ; p=.012\right)$ and WCST performance $\left(F_{1,61}=4.00 ; p=.048\right)$; however, mean differences were not found for the BRI variable $\left(F_{1,61}=0.57 ; p=.454\right)$.

\section{Exploring WCST as a Mediator Between Hippocampal Sclerosis and Depression}

Building on findings from the previous analysis, the degree to which WCST performance mediated the relationship between HS and depression was examined. The presence of HS was significantly associated with greater perseverative errors committed on the WCST $(\beta=-0.25 ; p=.048)$, which successfully predicted parental ratings of youth depression $(\beta=-0.41$; $p=<.001$ ). The previously significant relationship between children with HS and depression $(\beta=0.32 ; p=.012)$ was completely attenuated when WCST was included in the model ( $\beta=0.22 ; p=.068)$, thus fulfilling the requirements of Baron and Kenny's traditional causal steps approach for statistical mediation (Baron and Kenny, 1986). The significance of the mediation model was further confirmed by the $95 \%$ confidence interval range (95\% confidence interval $[\mathrm{CI}][0.12,15.81])$ derived by Preacher and Hayes' bootstrapping procedure for detecting indirect effects (Preacher \& Hayes, 2004). The results of these analyses are depicted in Figure 2.

\section{Examining the Impact of HS on Youth Depression}

To quantify the extent to which HS served as a specific risk factor for clinically elevated depression ratings, a logistic regression was conducted by dividing the sample according to those with or without HS. The presence of HS was 
Table 6. Summary of linear regression analysis for variables predicting depression in pediatric TLE

\begin{tabular}{lrrrrrrrr}
\hline \hline & \multicolumn{1}{c}{$B$} & \multicolumn{1}{c}{$\mathrm{S}_{\mathrm{E}}$} & $\beta$ & $p$-Value & Zero-order $r$ & Partial r & Semipartial r & Tolerance \\
\hline Constant & 53.37 & 18.04 & & & & & \\
Family Stress & 3.48 & 1.88 & 0.21 & 0.07 & 0.38 & 0.24 & 0.19 \\
BRI $^{*}$ & 0.33 & 0.15 & 0.29 & 0.03 & 0.50 & 0.28 & 0.24 & 0.72 \\
WCST $^{*}$ & -0.24 & 0.11 & -0.26 & 0.04 & -0.45 & -0.26 & -0.22 & 0.75 \\
\hline \hline
\end{tabular}

$\mathrm{BRI}=$ Behavioral Regulation Index TLE $=$ temporal lobe epilepsy; WCST $=$ Wisconsin Card Sorting Perseverative Errors. ${ }^{*} p<0.05$.

associated with a four-fold increased risk of clinically elevated depression ratings on the BASC-2 (odds ratio $=4.42$; $p=.014 ; 95 \%$ CI for odds ratio $[1.36,14.39])$.

\section{DISCUSSION}

Children and adolescents with TLE have been shown to be more susceptible to depressive symptoms than youth with other types of epilepsy (Salpekar et al., 2013; Schraegle \& Titus, 2017a; Titus et al., 2008). Given the deleterious impact of pediatric depressive symptoms on HRQOL (Clary, Vander Wal, \& Titus, 2010; Plioplys, 2003; Schraegle \& Titus, 2017a), the present study examined the unique contribution of sociodemographic, epilepsy specific, and neurocognitive factors on depressive features in children and adolescents with TLE. It is hoped that a more complete understanding of these factors will assist with early detection and screening for depression among children and adolescents with TLE.

With regard to the first research question, seizure-specific and sociodemographic factors were only marginally correlated with youth depressive features. For example, aside from a moderate association between HS and depressive symptoms, all other seizure-specific variables were only mildly associated with depressive symptoms. Despite research showing the adverse effect of seizure variables on HRQOL (Devinsky et al., 1999; Schraegle \& Titus, 2016), these factors were not strongly related to depressive features in our sample. Like seizure factors, most sociodemographic factors, such as maternal education, parental relationship status, and parental psychiatric history, were also marginally related to depressive symptoms. The exception to this was family stress, which was consistent with previous research (Dunn, Austin, \& Huster, 1999; Oostrom, Schouten, Kruitwagen, Peters \& Jennekens-Schinkel, 2001; Pianta \&

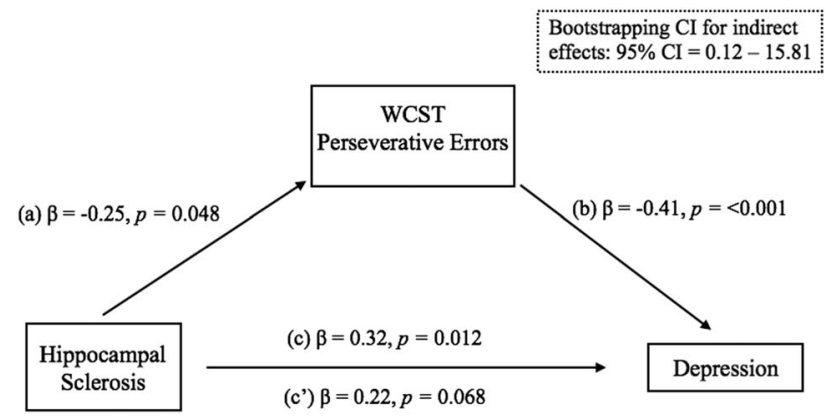

Fig. 2. Multiple linear regression analyses for statistical mediation.
Lothman, 1994). This highlights the important role of family stress on child adjustment in pediatric epilepsy.

The current study also demonstrated a significant relationship between cognitive functioning and depression features in youth with TLE. While this relationship was not observed across language, visuospatial reasoning, memory (verbal/ nonverbal), and attention domains, a significant relationship was specifically identified in the domain of executive functioning. That is, deficits in behavioral regulation (i.e., BRI) and cognitive flexibility (i.e., WCST perseverative errors) were significantly associated with higher depression ratings. This is a novel finding in a pediatric epilepsy population; however, research conducted with adult TLE samples has demonstrated the significant relationship between WCST perseverative responses and severity of self-reported depressive features (Seidenberg, Hermann, Noe, \& Wyler, 1995).

Espinosa et al. (2010) reported that greater levels of perseverative errors on the WCST were predictive of suicidality in adults with TLE. These findings suggest that depression symptoms may be related to underlying cognitive processes that regulate emotional and behavioral responses, indicating that youth with epilepsy who are experiencing greater executive dysfunction related to regulatory control may be at higher risk for depression. Moreover, by combining significant EF variables and family stress, it was further discovered that depression ratings were uniquely predicted both by the BRIEF BRI and the WCST perseverative errors variable. While not emerging as a significant predictor, a mild-to-moderate partial correlation was identified for family stress, suggesting an important but less prominent role for this variable.

While the mechanism of action between EF deficits and depression in pediatric TLE is unknown, prospective studies indicate that EF deficits play an active role in precipitating an initial depressive episode in adolescents (Letkiewicz et al., 2014). In non-clinical samples, trait negative affect has been associated with decreased activity in brain regions involved in top-down control of attention during emotional distraction and with disrupted top-down control of attention on a non-emotional task (Crocker et al., 2012). In this regard, EF deficits contribute to difficulties switching or shifting attention away from affective information, potentially leading to a prolonged focus on negative material. This, in turn, can maintain or increase negative affect and subsequently maintain or increase depression symptoms.

Joormann and Gotlib (2008) showed that prolonged focus on negative material could be associated with inhibition 
deficits, which can allow negative material to preoccupy working memory. EF deficits in cognitive flexibility may further serve to alter appraisal processes that may influence both physiological reactivity and subjective distress in response to adverse events. This can play a critical role in youth with epilepsy who, as a group, report higher levels of subjective distress (Chong et al., 2016). In the current sample, emotional control was found to be a significant predictor of depressive features in pediatric TLE. This may reflect an inability to modulate negative thoughts or fears related to epilepsy. When this modulation is poorly controlled, individuals with epilepsy may become overly engaged in emotional reactions and withdraw from goal directed behavior. This process would be compounded by inefficiencies in top-down cognitive control, such as the perseverative tendencies captured by the WCST.

The influence of family stress on depressive features in the present sample is not surprising when considering the elevated coping demands in this population. It is reasonable to expect that youth with epilepsy rely heavily on external resources, such as family, to manage the emotions that accompany their condition. However, when family stress is higher, family members, such as parents, may be less available or may even exacerbate maladaptive youth coping (Schraegle \& Titus, 2017b). It will be important for future research to investigate the extent to which family stress and other familial factors contribute to youth behavioral regulation in the context of depression in pediatric TLE. It could be that youth emotional control is partially predicted by parental adjustment, which may manifest in epilepsy shaming (Chong et al., 2016) and/or parental helplessness (McLaughlin, Schraegle, Nussbaum, \& Titus, 2016). Thus, adaptive coping in the face of epilepsy with depression is likely to be impacted by several factors. Determining these factors will be important for family based interventions tailored to treat depression among children and adolescents with TLE.

Similar to what has been previously found in the adult and pediatric TLE literature (Hermann \& Seidenberg, 1995; Rzezak et al., 2014; Sretton \& Thompson, 2012), the current study confirmed HS as a risk factor for EF deficits in youth with epilepsy. Specifically, youth with HS were found to have significantly more perseveration errors on the WCST than those without HS. In a sample of adults with TLE, Hermann and Seidenberg (1995) demonstrated that abnormal interictal discharge propagation on EEG from mesial temporal structures to the prefrontal cortex was associated with deficits observed on the WCST.

Analogous differences were not observed for parent rated behavioral regulation on the BRIEF. The presence of HS incurred a four-fold increase in the likelihood of clinically significant depressive features in our sample. Although this finding is often observed in the adult TLE literature (Quiske, Helmstaedter, Lux, \& Elger, 2000), to our knowledge, this work marks one of the first demonstrations of this relationship in a pediatric epilepsy sample. Of interest, the relationship between HS and depression was fully mediated by the WCST perseverative errors variable. This finding is important because it offers the first step toward developing a causal mechanism that comprehensively examines $\mathrm{HS}, \mathrm{EF}$, and emotional maladjustment. Longitudinal research is necessary to verify the directionality of these relationships.

Recently, Kemmotsu et al. (2013) demonstrated that depressive features in adult TLE was significantly associated with frontotemporal network dysfunction indicated by altered functional connectivity between the left hippocampus and left anterior prefrontal cortex (Brodmann area 10). Corcoran and Upton (1993) postulated that HS alone could explain executive dysfunction by compromising its role as a comparator of actions, wherein information about a previous response is used to guide future behavior via working memory.

Conversely, Hermann, Wyler, and Richey (1988) attributed EF deficits in TLE to frontal lobe dysfunction caused by propagation of "neural noise" from the temporal lobe, especially in the case of a mesial temporal seizure focus through pathways that connect the anterior temporal lobe and hippocampus with frontal regions. These two theories are now considered complimentary given that both short and longrange networks are altered by limbic system pathology. Of interest, similar morphological changes to the hippocampus, amygdala, and frontal networks are often observed in adults with MDD (Keller et al., 2009; Sheline, Gado, \& Kraemer, 2003). For this reason, depression and TLE has been described as an epiphenomenon (Valente \& Busatto Filho, 2013).

The significant and unique role of executive dysfunction in depression among youth with TLE elucidates another potential avenue for therapeutic intervention. Because sociodemographic and seizure-specific factors are not always modifiable, the potential of modifying depression risk or severity by targeting cognition, and specifically executive functioning, is intriguing. The presence of poor behavioral regulation as well as impaired cognitive flexibility and problem solving may be important in the development and maintenance of depression in this population.

The current work suggests a role for EF screening early in the condition to initiate intervention and/or minimize the potential for internalizing psychopathology. Because deficits in $\mathrm{EF}$ have also been found to be significant barriers for HRQOL in pediatric epilepsy (Schraegle \& Titus, 2016), attempting to modify these abilities may represent an area through which intervention could have a meaningful impact on functional outcomes. The types of executive dysfunction exhibited in the current study, namely emotional control and cognitive flexibility, provide insights into how cognitivebased interventions may improve coping and ultimately HRQOL in youth with TLE.

Some limitations to the present study should also be considered. All children and adolescents were referred for epilepsy treatment at a tertiary care center, which may reflect a greater severity of epilepsy. As such, these findings may be especially useful for those in similar settings, but may not generalize to those children and adolescents with less severe forms of TLE. The present study relied upon parent-report of depression features in children and adolescents with 
depression. This was done to avoid exclusion of patients of younger age, lower functioning, and higher epilepsy severity.

However, sole reliance on parent-report may have resulted in bias that should be clarified through future research. Moreover, family stress was examined by a single Likert item and dichotomized in our first analysis, which may have constricted sample variability. Future studies using more comprehensive rating measures of family stress are encouraged in future work on this topic. Lastly, morphological changes in the hippocampus were defined by the presence or absence of HS. Volumetric analyses were not conducted and, as such, the relationship of hippocampal volume with depression in pediatric TLE was not investigated.

The current study highlights and helps to define the strong association between executive functioning and parent rated depression in a sample of children and adolescents with TLE. While HS was found to be a predisposing factor for depression symptoms, EFs related to cognitive flexibility were found to mediate this relationship. Regular screening for executive dysfunction may help identify risk factors for depression to allow for prevention or early intervention. The current findings also suggest a potential mechanism for improving HRQOL outcomes and minimizing the risk for depression in youth with epilepsy by working to remediate EF deficits (e.g., Crocker et al., 2013). Intervention strategies aimed at improving emotional control and cognitive flexibility may improve effective coping strategies, which may in turn contribute to the prevention of depression and ultimately improve the lives of children and adolescents with TLE.

\section{ACKNOWLEDGEMENTS}

None of the authors received funding for this project. None of the authors have any conflicts of interest to disclose. We confirm that we have read the journal's position on issues involved in ethical publication and affirm that this report is consistent with those guidelines.

\section{REFERENCES}

Austin, J.K., Harezlak, J., Dunn, D.W., Huster, G.A., Rose, D.F., \& Ambrosius, W.T. (2001). Behavior problems in children before first recognized seizures. Pediatrics, 107(1), 115-122.

Baron, R.M., \& Kenny, D.A. (1986). The moderator-mediator variable distinction in social psychological research: Conceptual, strategic, and statistical considerations. Journal of Personality and Social Psychology, 51(6), 1173.

Campo, P., Maestu, F., Garcia-Morales, I., Gil-Nagel, A., Strange, B., Morales, M., \& Ortix, T. (2009). Modulation of medial temporal lobe activity in epilepsy patients with hippocampal sclerosis during verbal working memory. Journal of the International Neuropsychological Society, 15(4), 536-546.

Cataldo, M.G., Nobile, M., Lorusso, M.L., Battaglia, M., \& Molteni, M. (2005). Impulsivity in depressed children and adolescents: A comparison between behavioral and neuropsychological data. Psychiatry Research, 136(2), 123-133.

Chong, L., Jamieson, N.J., Gill, D., Singh-Grewal, D., Craig, J.C., Ju, A., ... Tong, A. (2016). Children's experiences of epilepsy: A systematic review of qualitative studies. Pediatrics, 138(3), e20160658.
Clary, L.E., Vander Wal, J.S., \& Titus, J.B. (2010). Examining health-related quality of life, adaptive skills, and psychological functioning in children and adolescents with epilepsy presenting for a neuropsychological evaluation. Epilepsy \& Behavior, 19(3), 487-493.

Corcoran, R., \& Upton, D. (1993). A role for the hippocampus in card sorting? Cortex, 29(2), 293-304.

Crocker, L.D., Heller, W., Warren, S.L., O'Hare, A.J., Infantolino, Z.P., \& Miller, G.A. (2013). Relationships among cognition, emotion, and motivation: Implications for intervention and neuroplasticity in psychopathology. Frontiers in Human Neuroscience, 7, 261.

Crocker, L.D., Heller, W., Spielberg, J.M., Warren, S.L., Bredemeier, K., Sutton, B.P., ... Miller, G.A. (2012). Neural mechanisms of attentional control differentiate trait and state negative affect. Frontiers in Psychology, 3, 298.

Davies, S., Heyman, I., \& Goodman, R. (2003). A population survey of mental health problems in children with epilepsy. Developmental Medicine and Child Neurology, 45(5), 292-295.

Delis, D.C., Kaplan, E., \& Kramer, J.H. (2001). Delis-Kaplan executive function system (D-KEFS). San Antonio, TX: Psychological Corporation.

Delis, D.C., Kramer, J.H., Kaplan, E., \& Ober, B.A. (1994). Manual for the California Verbal Learning Test-Children's Version. San Antonio, TX: Psychological Corporation.

Devinsky, O., Westbrook, L., Cramer, J., Glassman, M., Perrine, K., \& Camfield, C. (1999). Risk factors for poor health-related quality of life in adolescents with epilepsy. Epilepsia, 40(12), 1715-1720.

Dunn, D.W., Austin, J.K., \& Huster, G.A. (1999). Symptoms of depression in adolescents with epilepsy. Journal of the American Academy of Child \& Adolescent Psychiatry, 38(9), 1132-1138.

Ekinci, O., Titus, J.B., Rodopman, A.A., Berkem, M., \& Trevathan, E. (2009). Depression and anxiety in children and adolescents with epilepsy: Prevalence, risk factors, and treatment. Epilepsy \& Behavior, 14(1), 8-18.

Engel, J. (2001). A proposed diagnostic scheme for people with epileptic seizures and with epilepsy: Report of the ILAE Task Force on Classification and Terminology. Epilepsia, 42(6), 796-803.

Espinosa, A.G., Machado, R.A., González, S.B., González, M.E.G., Montoto, A.P., \& Sotomayor, G.T. (2010). Wisconsin Card Sorting Test performance and impulsivity in patients with temporal lobe epilepsy: Suicidal risk and suicide attempts. Epilepsy \& Behavior, 17(1), 39-45.

Focke, N.K., Yogarajah, M., Bonelli, S.B., Bartlett, P.A., Symms, M.R., \& Duncan, J.S. (2008). Voxel-based diffusion tensor imaging in patients with mesial temporal lobe epilepsy and hippocampal sclerosis. Neuroimage, 40(2), 728-737.

Gilliam, F.G., Maton, B.M., Martin, R.C., Sawrie, S.M., Faught, R. E., Hugg, J.W., \& Kuzniecky, R.I. (2007). Hippocampal 1HMRSI correlates with severity of depression symptoms in temporal lobe epilepsy. Neurology, 68(5), 364-368.

Gioia, G.A., Isquith, P.K., Guy, S.C., \& Kenworthy, L. (2000). Behavior Rating Inventory of Executive Function: BRIEF. Odessa, FL: Psychological Assessment Resources.

Gottlieb, L., Zelko, F.A., Kim, D.S., \& Nordli, D.R. (2012). Cognitive proficiency in pediatric epilepsy. Epilepsy \& Behavior, 23(2), 146-151.

Gyurak, A., Goodkind, M.S., Kramer, J.H., Miller, B.L., \& Levenson, R.W. (2012). Executive functions and the downregulation and up-regulation of emotion. Cognition \& Emotion, 26(1), 103-118. 
Heaton, R.K., Chelune, G.J., Talley, J.L., Kay, G.G., \& Curtiss, G. (1993). Wisconsin card sorting test manual, revised and expanded. Lutz, FL: Psychological Assessment Resources, Inc.

Hermann, B., \& Seidenberg, M. (1995). Executive system dysfunction in temporal lobe epilepsy: Effects of nociferous cortex versus hippocampal pathology. Journal of Clinical and Experimental Neuropsychology, 17(6), 809-819.

Hermann, B.P., Seidenberg, M., \& Bell, B. (2002). The neurodevelopmental impact of childhood onset temporal lobe epilepsy on brain structure and function and the risk of progressive cognitive effects. Progress in Brain Research, 135, 429-438.

Hermann, B.P., Wyler, A.R., \& Richey, E.T. (1988). Wisconsin Card Sorting Test performance in patients with complex partial seizures of temporal-lobe origin. Journal of Clinical and Experimental Neuropsychology, 10(4), 467-476.

Holler, K., Kavanaugh, B., \& Cook, N.E. (2014). Executive functioning in adolescent depressive disorders. Journal of Child and Family Studies, 23(8), 1315-1324.

Igarashi, K., Oguni, H., Osawa, M., Awaya, Y., Kato, M., Mimura, M., \& Kashima, H. (2002). Wisconsin card sorting test in children with temporal lobe epilepsy. Brain and Development, 24(3), 174-178.

Joormann, J., \& Gotlib, I.H. (2008). Updating the contents of working memory in depression: Interference from irrelevant negative material. Journal of Abnormal Psychology, 117(1), 182.

Kaiser, R.H., Andrews-Hanna, J.R., Wager, T.D., \& Pizzagalli, D.A. (2015). Large-scale network dysfunction in major depressive disorder: A meta-analysis of resting-state functional connectivity. JAMA Psychiatry, 72(6), 603-611.

Kaplan, E., Goodglass, H., \& Weintraub, S. (2001). Boston naming test. Austin, TX: Pro-Ed.

Kavanaugh, B.C., Scarborough, V.R., \& Salorio, C.F. (2015). Parent-rated emotional-behavioral and executive functioning in childhood epilepsy. Epilepsy \& Behavior, 42, 22-28.

Keller, S.S., Baker, G., Downes, J.J., \& Roberts, N. (2009). Quantitative MRI of the prefrontal cortex and executive function in patients with temporal lobe epilepsy. Epilepsy \& Behavior, 15(2), 186-195.

Kemmotsu, N., Kucukboyaci, N.E., Cheng, C.E., Girard, H.M., Tecoma, E.S., Iragui, V.J., \& McDonald, C.R. (2013). Alterations in functional connectivity between the hippocampus and prefrontal cortex as a correlate of depressive symptoms in temporal lobe epilepsy. Epilepsy \& Behavior, 29(3), 552-559.

Klimkeit, E.I., Tonge, B., Bradshaw, J.L., Melvin, G.A., \& Gould, K. (2011). Neuropsychological deficits in adolescent unipolar depression. Archives of Clinical Neuropsychology, 26(7), 662-676.

Ladouceur, C.D., Dahl, R.E., Williamson, D.E., Birmaher, B., Ryan, N.D., \& Casey, B.J. (2005). Altered emotional processing in pediatric anxiety, depression, and comorbid anxiety-depression. Journal of Abnormal Child Psychology, 33(2), 165-177.

Letkiewicz, A.M., Miller, G.A., Crocker, L.D., Warren, S.L., Infantolino, Z.P., Mimnaugh, K.J., \& Heller, W. (2014). Executive function deficits in daily life prospectively predict increases in depressive symptoms. Cognitive Therapy and Research, 38(6), 612-620.

Lopes, A.F., Simoes, M.M., Robalo, C.N., Fineza, I., \& Gonçalves, O.B. (2010). Neuropsychological evaluation in children with epilepsy: Attention and executive functions in temporal lobe epilepsy. Revista de neurologia, 50(5), 265-272.

McLaughlin, R., Schraegle, W., Nussbaum, N., \& Titus, J.B. (2016). Parental feelings of helplessness and internalizing psychopathology are unique predictors of health-related quality of life in pediatric epilepsy. AES Abstracts, 2, 343.

Oguz, A., Kurul, S., Dirik, E., \& Eylül, D. (2002). Relationship of epilepsy-related factors to anxiety and depression scores in epileptic children. Journal of Child Neurology, 17(1), 37-40.

Oostrom, K.J., Schouten, A., Kruitwagen, C.L., Peters, A.C., \& Jennekens-Schinkel, A. (2001). Parents' perceptions of adversity introduced by upheaval and uncertainty at the onset of childhood epilepsy. Epilepsia, 42(11), 1452-1460.

Pianta, R.C., \& Lothman, D.J. (1994). Predicting behavior problems in children with epilepsy: Child factors, disease factors, family stress, and child-mother interaction. Child Development, 65(5), 1415-1428.

Plioplys, S. (2003). Depression in children and adolescents with epilepsy. Epilepsy \& Behavior, 4, 39-45.

Preacher, K.J., \& Hayes, A.F. (2004). SPSS and SAS procedures for estimating indirect effects in simple mediation models. Behavior Research Methods, Instruments, \& Computers, 36(4), 717-731.

Quiske, A., Helmstaedter, C., Lux, S., \& Elger, C.E. (2000). Depression in patients with temporal lobe epilepsy is related to mesial temporal sclerosis. Epilepsy Research, 39(2), 121-125.

Reynolds, C.R., \& Kamphaus, R.W. (2004). BASC-2: Behavior assessment system for children (2nd ed.), Circle Pines, MN: American Guidance Service.

Reynolds, C.R., \& Voress, J.K. (2007). Test of memory and learning (2nd ed.). Austin, TX: Pro-Ed.

Richardson, E.J., Griffith, H.R., Martin, R.C., Paige, A.L., Stewart, C.C., Jones, J., ... Seidenberg, M. (2007). Structural and functional neuroimaging correlates of depression in temporal lobe epilepsy. Epilepsy \& Behavior, 10(2), 242-249.

Rodenburg, R., Stams, G.J., Meijer, A.M., Aldenkamp, A.P., \& Deković, M. (2005). Psychopathology in children with epilepsy: A meta-analysis. Journal of Pediatric Psychology, 30(6), 453-468.

Rutter, M., Graham, P.J., \& Yule, W. (1970). A neuropsychiatric study in childhood (No. 6). London: Heinemann Educational Books.

Rzezak, P., Fuentes, D., Guimarães, C.A., Thome-Souza, S., Kuczynski, E., Guerreiro, M., ... Valente, K.D. (2009). Executive dysfunction in children and adolescents with temporal lobe epilepsy: Is the Wisconsin Card Sorting Test enough? Epilepsy \& Behavior, 15(3), 376-381.

Rzezak, P., Fuentes, D., Guimarães, C.A., Thome-Souza, S., Kuczynski, E., Li, L.M., \& Valente, K.D. (2007). Frontal lobe dysfunction in children with temporal lobe epilepsy. Pediatric Neurology, 37(3), 176-185.

Rzezak, P., Valente, K.D., \& Duchowny, M.S. (2014). Temporal lobe epilepsy in children: Executive and mnestic impairments. Epilepsy \& Behavior, 31, 117-122.

Salpekar, J.A., Berl, M.M., Havens, K., Cushner-Weinstein, S., Conry, J.A., Pearl, P.L., \& Gaillard, W.D. (2013). Psychiatric symptoms in children prior to epilepsy surgery differ according to suspected seizure focus. Epilepsia, 54(6), 1074-1082.

Schraegle, W.A., \& Titus, J.B. (2016). Executive function and health-related quality of life in pediatric epilepsy. Epilepsy \& Behavior, 62, 20-26.

Schraegle, W.A., \& Titus, J.B. (2017a). The relationship of seizure focus with depression, anxiety, and health-related quality of life in children and adolescents with epilepsy. Epilepsy \& Behavior, 68, 115-122.

Schraegle, W.A., \& Titus, J.B. (2017b). The influence of endophenotypic, disease-specific, and environmental variables on the expression of anxiety in pediatric epilepsy. Epilepsy \& Behavior, 75, 90-96. 
Sheline, Y.I. (2003). Neuroimaging studies of mood disorder effects on the brain. Biological Psychiatry, 54(3), 338-352.

Sheline, Y.I., Gado, M.H., \& Kraemer, H.C. (2003). Untreated depression and hippocampal volume loss. American Journal of Psychiatry, 160(8), 1516-1518.

Seidenberg, M., Hermann, B., Noe, A., \& Wyler, A.R. (1995). Depression in temporal lobe epilepsy: Interaction between laterality of lesion and Wisconsin Card Sort Performance. Neuropsychiatry, Neuropsychology, \& Behavioral Neurology, 8(2), 81-87.

Stretton, J., \& Thompson, P.J. (2012). Frontal lobe function in temporal lobe epilepsy. Epilepsy Research, 98(1), 1-13.

Thome-Souza, S., Kuczynski, E., Assumpção, F., Rzezak, P., Fuentes, D., Fiore, L., \& Valente, K.D. (2004). Which factors may play a pivotal role on determining the type of psychiatric disorder in children and adolescents with epilepsy? Epilepsy \& Behavior, 5(6), 988-994.
Titus, J.B., Kanive, R., Sanders, S.J., \& Blackburn, L.B. (2008). Behavioral profiles of children with epilepsy: Parent and teacher reports of emotional, behavioral, and educational concerns on the BASC-2. Psychology in the Schools, 45(9), 893-904.

Valente, K.D., \& Busatto Filho, G. (2013). Depression and temporal lobe epilepsy represent an epiphenomenon sharing similar neural networks: Clinical and brain structural evidences. Arquivos de neuro-psiquiatria, 71(3), 183-190.

Wagner, S., Müller, C., Helmreich, I., Huss, M., \& Tadić, A. (2015). A meta-analysis of cognitive functions in children and adolescents with major depressive disorder. European Child \& Adolescent Psychiatry, 24(1), 5-19.

Wechsler, D. (2003). Wechsler intelligence scale for childrenWISC-IV. San Antonio, TX: Psychological Corporation. 